\title{
Secondary Central Chondrosarcoma
}

National Cancer Institute

\section{Source}

National Cancer Institute. Secondary Central Chondrosarcoma. NCI Thesaurus. Code C121881.

A chondrosarcoma that arises in a pre-existing enchondroma. 